\title{
Las bibliotecas universitarias y Facebook: cómo y por qué estar presentes
}

\author{
Por Dídac Margaix-Arnal
}

\begin{abstract}
Resumen: Durante el último año la popularidad de los sitios de redes sociales ha aumentado considerablemente en nuestro país. Su presencia en la vida cotidiana de nuestros usuarios, el número creciente de miembros de estas redes y la aparición de aplicaciones bibliotecarias ha despertado el interés de los profesionales de la información. En este artículo se analizan el concepto y las tipologías de los sitios de redes sociales, se plantean los criterios para seleccionar una red social, se justifica la elección de Facebook para una biblioteca universitaria y la creación de páginas como modelo de presencia más adecuado para una biblioteca. En la última parte del trabajo se analiza cómo crear y promocionar una página Facebook y cómo organizar eventos en ese sitio web.

Palabras clave: Sitios de redes sociales, Redes sociales, Facebook, Bibliotecas universitarias.

Title: Academic libraries and Facebook: how and why we should be there

Abstract: Over the past year, the popularity of social networking sites has seen tremendous -growth in Spain. Because of these networks' everyday online ubiquity, the rising number of new users, and the integration of library applications, information professionals have taken a huge interest in social networking sites. This article analyzes the concept behind social networking sites, different types of these sites, the use of Facebook as an academic library, and the creation of Facebook pages as the best presence model for libraries. The final part of the article discusses how to create and promote a Facebook page and how to organize events on the web site.

Keywords: Social network sites, Social networks, Facebook, Academic libraries.

Margaix-Arnal, Dídac. "Las bibliotecas universitarias y Facebook: cómo y por qué estar

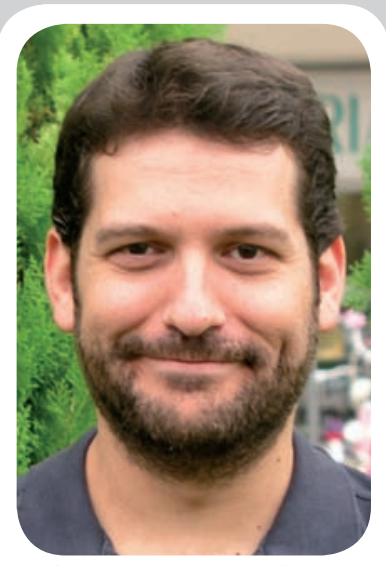

Dídac Margaix-Arnal, diplomado en biblioteconomía y documentación por la Universidad de Valencia, trabaja actualmente en la Biblioteca General de la Universidad Politécnica de Valencia. Ha impartido cursos de formación a bibliotecarios sobre nuevas tecnologías, publicado artículos y participado en congresos nacionales e internacionales sobre la web 2.0 y la biblioteca 2.0 . Edita el blog DosPuntoCero. presentes". En: El profesional de la información, 2008, noviembre-diciembre, v. 17, n. 6, pp. 589-601.
\end{abstract}

DOI: 10.3145/epi.2008.nov.02

\section{Introducción}

La web 2.0 ha sido un fenómeno muy estudiado en los últimos años. Si los blogs fueron su primer elemento destacable, en 2008 ha habido un importante aumento de la utilización, difusión y estudio de los sitios de redes sociales (en adelante SRS). La traducción a diferentes lenguas de la interfaz de algunos de estos sitios, su creciente número de miembros y la creación de APIs (application programming interfaces) que permiten a usuarios y empresas externas desarrollar sus propias aplicaciones, han contribuido probablemente a esta popularización.

En cualquier caso, en el último año los SRS han aumentado el número de integrantes, el volumen de tráfico de internet y la popularidad. Han despertado interés tanto entre la población en general como entre los profesionales de la información. Éstos ven las redes sociales como una nueva forma de obtener visibilidad y ofrecer servicios a sus usuarios, lo que se ha plasmado en numerosas publicaciones (Charnigo, 2007; Mack, 2007; Miller, 2007; Mitchell, 2007; Axelsson, 2008; Arroyo, 2008a; Behrens, 2008).

En este artículo se presenta una de las múltiples formas de presencia y visibilidad que ofrecen estas redes. Aunque este trabajo se basa en un SRS en concreto como es Facebook y hace referencia esencialmente a las bibliotecas universitarias, las ideas planteadas pueden ser aplicadas a otros tipos de bibliotecas y a otros SRS realizando los ajustes oportunos. 


\section{"En 2008 los sitios de redes sociales} han aumentado el número de usuarios, el volumen de tráfico de internet y la popularidad"

\section{El concepto de sitios de redes sociales}

Cada ser humano se relaciona con una serie de personas, las cuales establecen a su vez vínculos con otras. Nosotros, nuestros amigos y los amigos de nuestros amigos forman nuestra propia red social. Como consecuencia de esta realidad surgen los SRS. Los autores que han analizado este concepto (Boyd, 2007; Arroyo, 2008a) los definen como "servicios web que permiten a los individuos crear un perfil público o semi-público dentro de una plataforma en línea, articular una lista de otros usuarios con los cuales comparten algún tipo de contacto, así como ver y navegar entre su lista de contactos y los que éstos han realizado dentro del sistema". Arroyo (2008a) destaca también las posibilidades de comunicación y de compartir objetos multimedia.

Los SRS se pueden clasificar siguiendo varios criterios. Farkas (2007) los diferencia en función de la edad de sus integrantes, y Arroyo (2008b) según las motivaciones del usuario o los propósitos del SRS, considerando tres grupos: sitios de carácter general, orientados a temas personales o de ocio, donde destacan MySpace, Facebook, Hi5 o Tuenti, este último muy popular entre los universitarios españoles; sitios profesionales, orientados a la actividad laboral y empresarial, representados por LinkedIn o Xing; y SRS especializados, como nVivo sobre conciertos o Vivu para profesionales de la salud. La división entre SRS de propósito general y profesionales también es seguida por Fumero y García (2008).

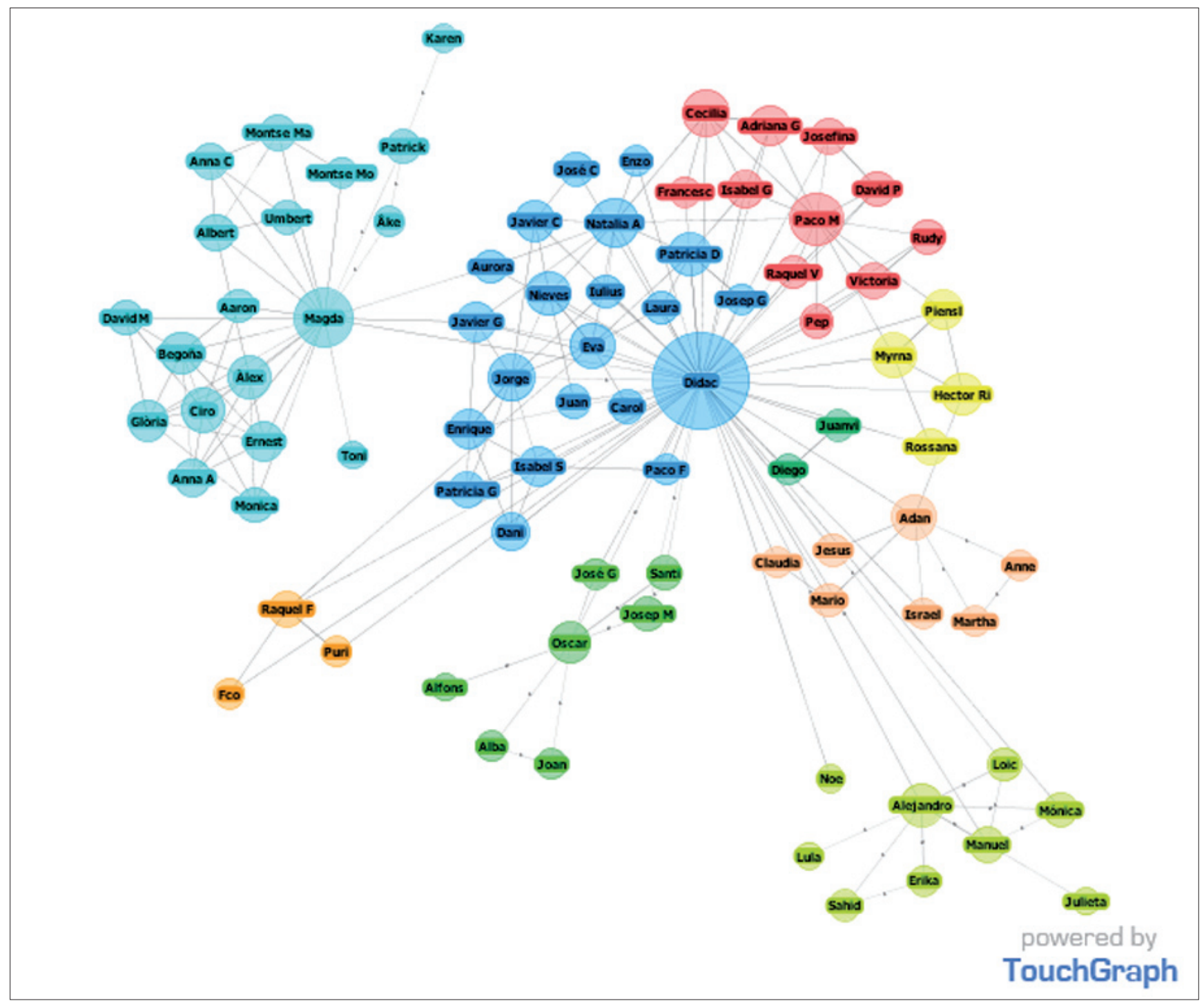

Representación de la red social de un individuo. Puede observarse cómo la red está formada por varias personas que se organizan en grupos compactos entre sí, pero con pocas relaciones con otros grupos 
En la actualidad tienden a convertirse en plataformas abiertas con funcionalidades y aplicaciones aportadas por el sistema y herramientas que permiten a usuarios y otras empresas desarrollar nuevas aplicaciones. Este amplio y flexible conjunto de recursos, unido al hecho de que el contenido es generado por personas o instituciones que presentan algún tipo de vínculo relevante para el usuario, ha aumentado el atractivo de los SRS.

Por este motivo las bibliotecas han comenzado a plantearse su presencia en estos sitios: para estar donde los usuarios están, utilizar las mismas plataformas y canales de comunicación que ellos y seguir siendo relevantes en el contexto de su experiencia del uso de internet.

\section{"En la actualidad los SRS tienden a convertirse en plataformas abiertas"}

\section{Seleccionar un SRS}

El gran número de servicios disponibles hace que sea difícil mantener un perfil actualizado en todos, por lo que es conveniente establecer una estrategia y seleccionar un SRS que sea adecuado para la biblioteca. El criterio principal de elección debe ser el de las preferencias de los usuarios del centro. Es decir, en principio se ha de optar por el que ellos hayan seleccionado mayoritariamente para relacionarse entre sí. Otra pauta a tener en cuenta es el objetivo del sitio y las expectativas de los usuarios. No es conveniente elaborar la presencia de la biblioteca en redes utilizadas exclusivamente para los contactos personales, pues los miembros lo pueden percibir como una intromisión en su privacidad y tener un efecto contrario al deseado (JISC, 2007).

En el caso de España, el estudio más reciente (Universal McCann, 2008) cifra en un $43,2 \%$ los internautas

\section{Sitios de redes sociales}

- Facebook.

http://www.facebook.com

- Hi5.

http://www.hi5.com/

- Linkedln. http://www.linkedin.com/

- MySpace. http://www.myspace.com/

- nVivo. http://www.nvivo.es/

- Tuenti. http://www.tuenti.com

- Vivu. http://vi.vu/

- Xing. http://www.xing.com/

españoles con perfil en alguna red social. Este dato representaría un $19,7 \%$ de los españoles entre 16 y 54 años, aproximadamente 4.700 .000 personas. Según el ranking general de Alexa ${ }^{1}$-con todas las webs-el SRS más utilizado en España es Tuenti en el décimo lugar, seguido de Facebook en el puesto 12, Hi5 en el 22 y

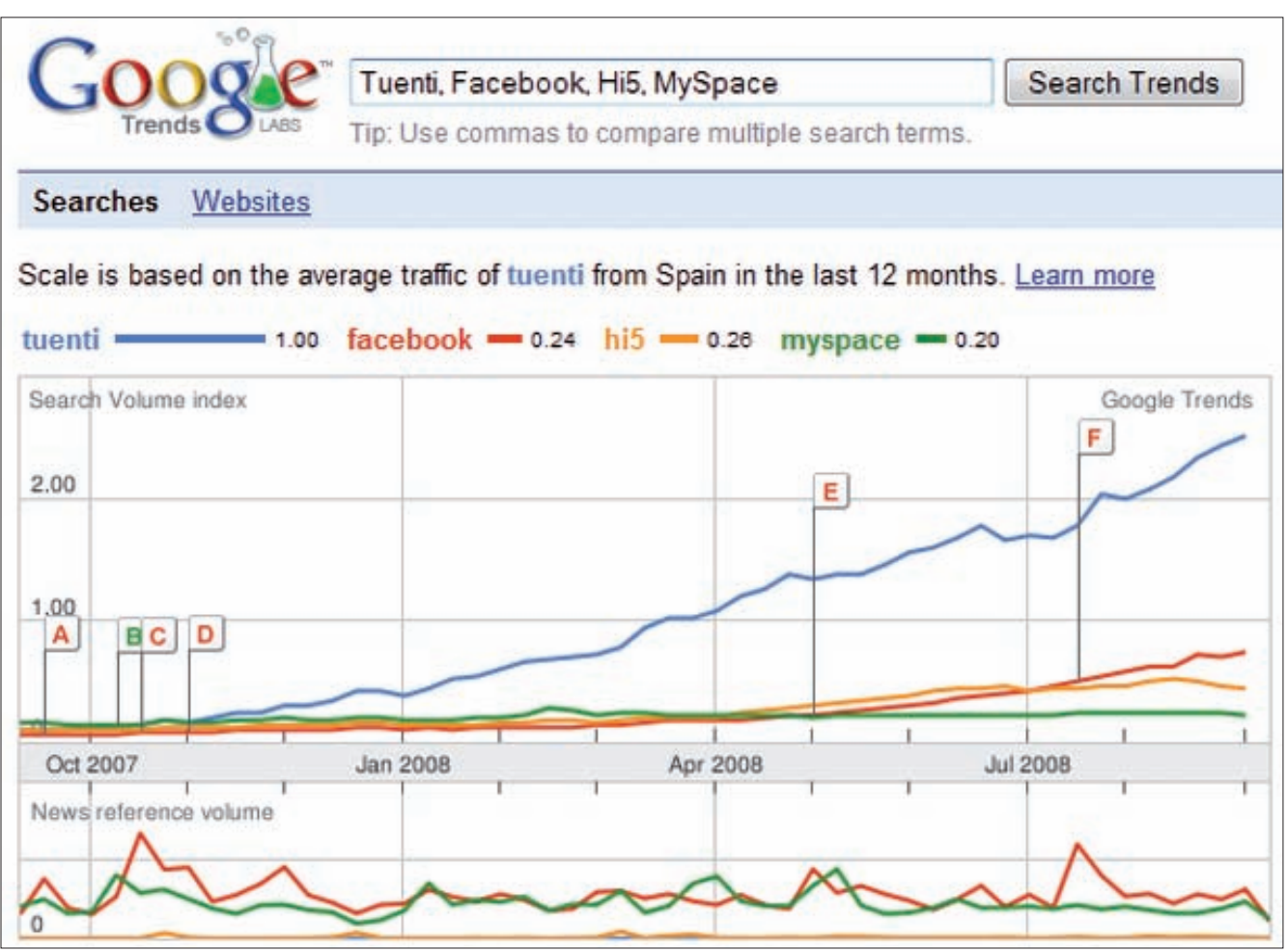

Gráfico de las búsquedas asignadas a España en los últimos 12 meses de los términos Tuenti, Facebook, Hi5 y MySpace. Fuente: Google Trends. Consultado en: 18-09-08 
MySpace en el 26 (consulta realizada el día 19-09-08). Si se complementan estos datos con una comparativa en Google Trends ${ }^{2}$ en la misma fecha y asumiendo que el número de búsquedas es un indicador del grado de uso de estos sitios, se observa que el ranking de búsquedas coincide con el de Alexa y que el fenómeno ha empezado a ser más relevante en los últimos 12 meses.

A pesar de que Tuenti es la red social más utilizada en España, si se tiene en cuenta que sólo se puede acceder mediante invitación de un miembro, que el número de invitaciones por usuario es limitado y que carece de herramientas para crear aplicaciones, son factores que limitan su interés para la biblioteca. MySpace en cambio tiene un importante peso de la cultura musical y artística. Hi5 por su parte tiene un gran número de usuarios jóvenes, pero está mucho más orientado a las relaciones personales. Por último Facebook tiene una significativa presencia de usuarios que son estudiantes universitarios (de hecho el sitio web se originó en la Harvard University), y cuenta con una importante API para construir aplicaciones. Los estudiantes han incluido Facebook tanto en sus rutinas de trabajo como en las relaciones personales (Thelwall, 2008). Se puede hablar por tanto de públicos distintos y cada biblioteca deberá evaluar cuál es el mejor canal para llegar a sus usuarios, pero parece que la mayoría de bibliotecas universitarias han optado por Facebook mientras que las públicas apuestan por MySpace (Primary Research Group, 2008).

Facebook es interesante por su facilidad de uso y por las herramientas de que dispone para crear aplicaciones. Ha conseguido formar una amplia comunidad de desarrolladores que han puesto a disposición de los miembros las aplicaciones más diversas: para recomendar lecturas, saludar de formas divertidas, representar las redes de contactos o buscar en catálogos bibliográficos, por citar sólo algunos ejemplos.

En este trabajo se recomienda la presencia en Facebook, pese a no ser la red social más utilizada (y vistas las restricciones de Tuenti) porque: tiene un considerable número de miembros; posee una cultura universitaria que permite a la biblioteca encontrar su espacio más fácilmente; está más abierta a nuevos usuarios; presenta una gran cantidad de aplicaciones disponibles; y existen muchas posibilidades de desarrollar otras nuevas que se ajusten a las necesidades de la biblioteca. Algunos estudios han demostrado la capacidad de Facebook para generar y mantener el capital social de sus miembros (Ellison, 2007), algo esencial si se desea que éstos participen en los servicios de la biblioteca.

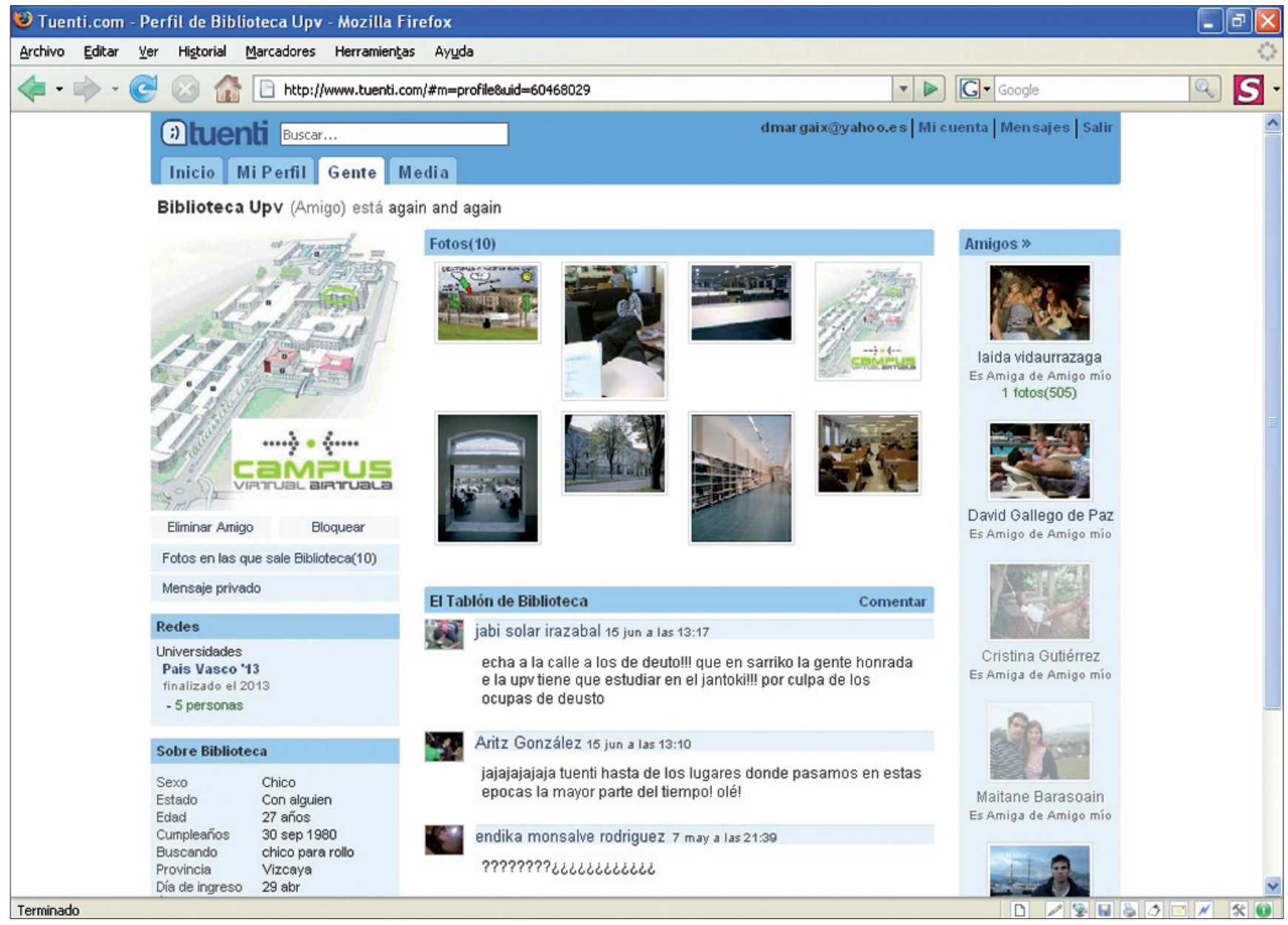

Perfil en Tuenti de la biblioteca de la Universidad del País Vasco-Euskal Herriko Unibertsitatea 
Es fácil observar cómo muchos estudiantes tienen sus perfiles en Facebook vinculados a dos universidades: una española y otra extranjera. Esto es debido a que muchos de ellos se registran cuando vuelven de estancias en el extranjero para mantener el contacto con sus compañeros. También es considerable la presencia de estudiantes extranjeros que participan en los programas de intercambio académico; si utilizan esta red para iniciar los contactos con su universidad de acogida, ésta puede ser una buena plataforma para que la biblioteca se dé a conocer entre los nuevos usuarios.

\section{"Facebook puede ser una buena plataforma para que la biblioteca de la universidad se dé a conocer entre los nuevos estudiantes extranjeros"}

\section{Modelos de presencia en Facebook}

Una vez decidida la presencia en un SRS, hay que plantearse cómo incluir a la biblioteca en ese tejido social. Las herramientas que ofrece Facebook para es- tar presentes son básicamente tres: perfiles, grupos de usuarios y páginas.

\subsection{Perfiles}

Son las piezas fundamentales y las más flexibles. Cada usuario crea el suyo propio con datos personales, fotografías, etc. Posteriormente puede unirse a una o varias redes, las cuales suelen representar áreas geográficas, universidades, empresas importantes, etc. Es posible personalizar cada perfil instalando aplicaciones con las más diversas funcionalidades y estableciendo el nivel de privacidad que se crea conveniente.

Las principales ventajas de usar los perfiles para la biblioteca son la existencia de muchas aplicaciones disponibles, que son la forma habitual de presencia en esta red social y que tienen más funcionalidades de comunicación con otros usuarios: mensajes, chats y comentarios. Por ello no es de extrañar que muchas bibliotecas opten por ellos.

Pero también tienen inconvenientes. Uno es que nunca están disponibles para usuarios no registrados en Facebook, y otro es que en cierta ocasión los perfiles de bibliotecas fueron eliminados por los administradores de Facebook por "no corresponder con nombres reales de personas", por lo que sería conveniente pensar

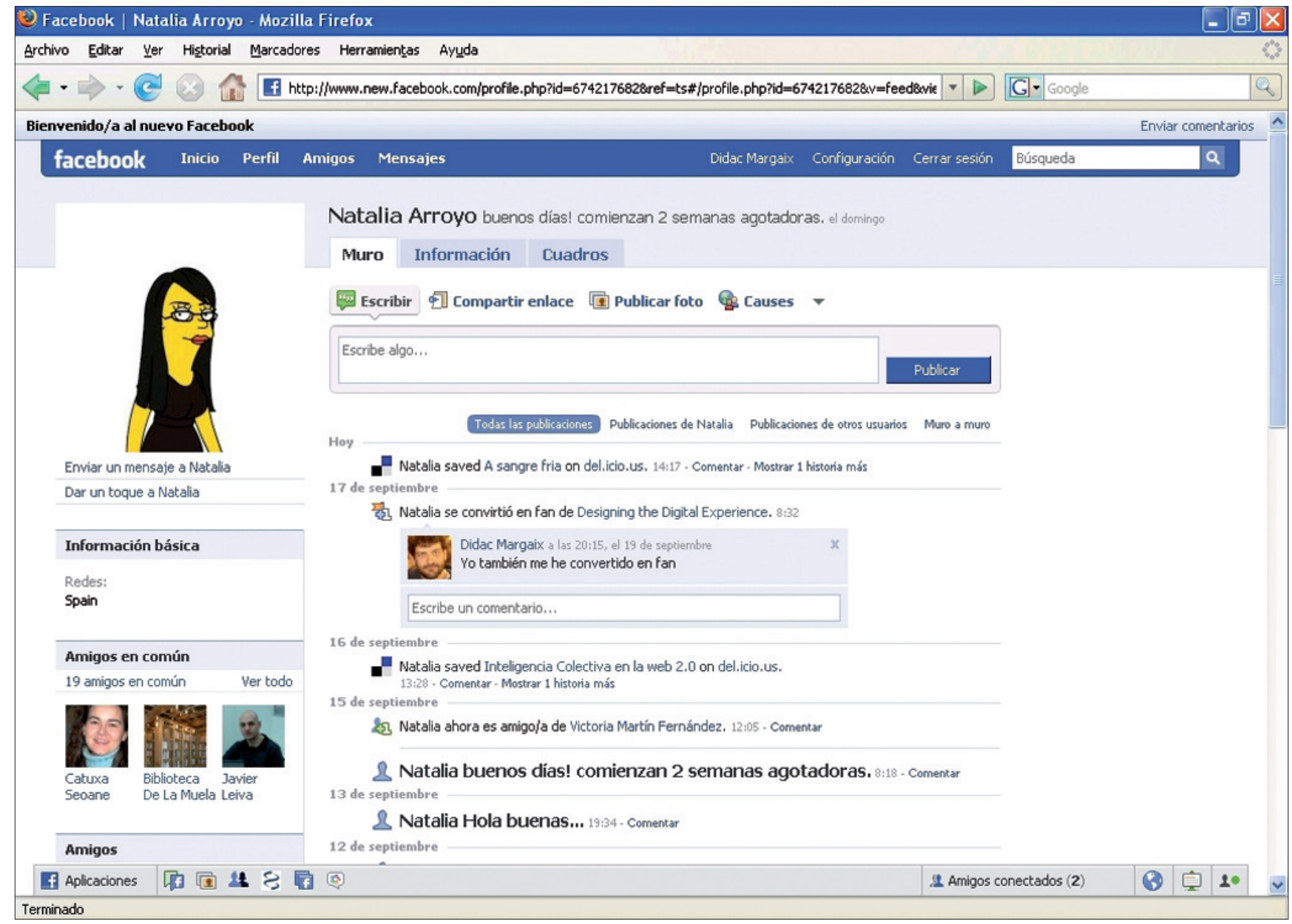

Perfil en Facebook de Natalia Arroyo. Puede verse la actividad reciente recogida en la plataforma y los comentarios de otros usuarios 
en otras formas más seguras de presencia (Behrens, 2008).

Si los perfiles están pensados para personas es mejor dejar esta opción. De cualquier forma la biblioteca puede estar presente a través de los perfiles de su personal, es decir, la presencia del director o directora de la biblioteca, el personal de referencia o quien se estime oportuno.

\subsection{Grupos}

Muchas bibliotecas han optado por crear grupos. Estos pueden ser creados de forma gratuita por cualquier usuario y otras personas pueden unirse por iniciativa propia o mediante invitación de alguno de sus miembros.

Entre sus ventajas están su facilidad de creación, las posibilidades de promoción al poder invitar a los contactos a unirse a él y una opción muy importante que permite a los administradores enviar mensajes a todos los miembros. El principal inconveniente es que sólo pueden tener las aplicaciones básicas: foros de discusión, muro de mensajes, publicación de fotografías, etc.

\subsection{Páginas}

Son la forma de presencia que el sitio recomienda para empresas, personajes famosos y otras organizaciones. Su lanzamiento se produjo en noviembre de 2007 y desde entonces unas 500 bibliotecas han optado por construir las suyas con distintos niveles de éxito (Behrens, 2008). Su aspecto es similar al de un perfil, pero con funciones distintas. La utilización de páginas frente a perfiles o grupos tiene una serie de ventajas y de inconvenientes.

\section{a. Ventajas:}

- Se puede personalizar su contenido instalando aplicaciones.

- Están pensadas para la presencia de organizaciones, por lo que tienen algunas herramientas más adecuadas.

- Su contenido está accesible para usuarios externos a la red, no es preciso registrarse para verlo.

- Los usuarios del SRS pueden registrarse libremente como seguidores o fans de estas páginas.

- Existe un apartado de estadísticas que aporta información sobre los accesos y la actividad de los usuarios en la página.

- Dispone de herramientas para comunicar novedades importantes a los usuarios, que aparecen como "Actualizaciones" en su perfil. Es un sistema menos visible que los mensajes, pero también menos intrusivo y por tanto generará menos rechazo entre los usuarios.
- La página puede tener como administradores a varios usuarios de Facebook.

- Son gratuitas.

- Dan a la organización una imagen más respetable y más visibilidad (Vander Veer, 2008).

\section{b. Inconvenientes:}

- Menor número de aplicaciones disponibles que los perfiles.

- Difícil promoción. No cuentan con herramientas propias para darse a conocer y no se puede invitar a otro usuario a hacerse seguidor de una página. Su promoción va a requerir algo de esfuerzo.

- No se reciben avisos de la actividad de los usuarios en la página (anotaciones en el muro, mensajes en el foro, etc.) por lo que se tendrán que supervisar estos canales para responder a las preguntas de los usuarios.

- No pueden ejercer como "actores" del SRS. En una página no se pueden realizar anotaciones en muros ajenos, ni comentar las fotos o los estados de otros usuarios, etc.

\section{Construcción de una página en Facebook}

Es conveniente que antes de iniciar este proceso la persona o personas que vayan a encargarse de mantenerla utilicen la plataforma durante algún tiempo. Esta fase previa de entrenamiento permitirá conocer su funcionamiento técnico así como la forma en que los miembros se relacionan dentro de ella. Sin esa información es muy probable que la página no encaje en la estructura y en la cultura del SRS y termine convirtiéndose en algo infrautilizado.

Como la mayoría de sitios de la web 2.0, Facebook aplica el principio de "beta perpetuo". Esto hace que esté permanentemente introduciendo pequeñas novedades en su interfaz por lo que algunos de los contenidos de este apartado pueden variar en breve espacio de tiempo.

\subsection{Objetivo de la página}

La primera decisión será establecer qué se quiere conseguir: puede que sea facilitar a los usuarios un canal más de comunicación, crear una presencia de la biblioteca para mantener actualizada su imagen ante los usuarios más jóvenes, establecer un punto de inicio de la conversación, etc. Las bibliotecas suelen ofrecer en el SRS su información de contacto, localización, horarios, consulta del catálogo, enlaces más relevantes, etc. Es decir, las páginas se constituyen en pequeñas sucursales digitales, presentando un acceso rápido y directo para aquellas personas que ya tienen actividad en esa red social. Sería poco razonable por tanto plan- 


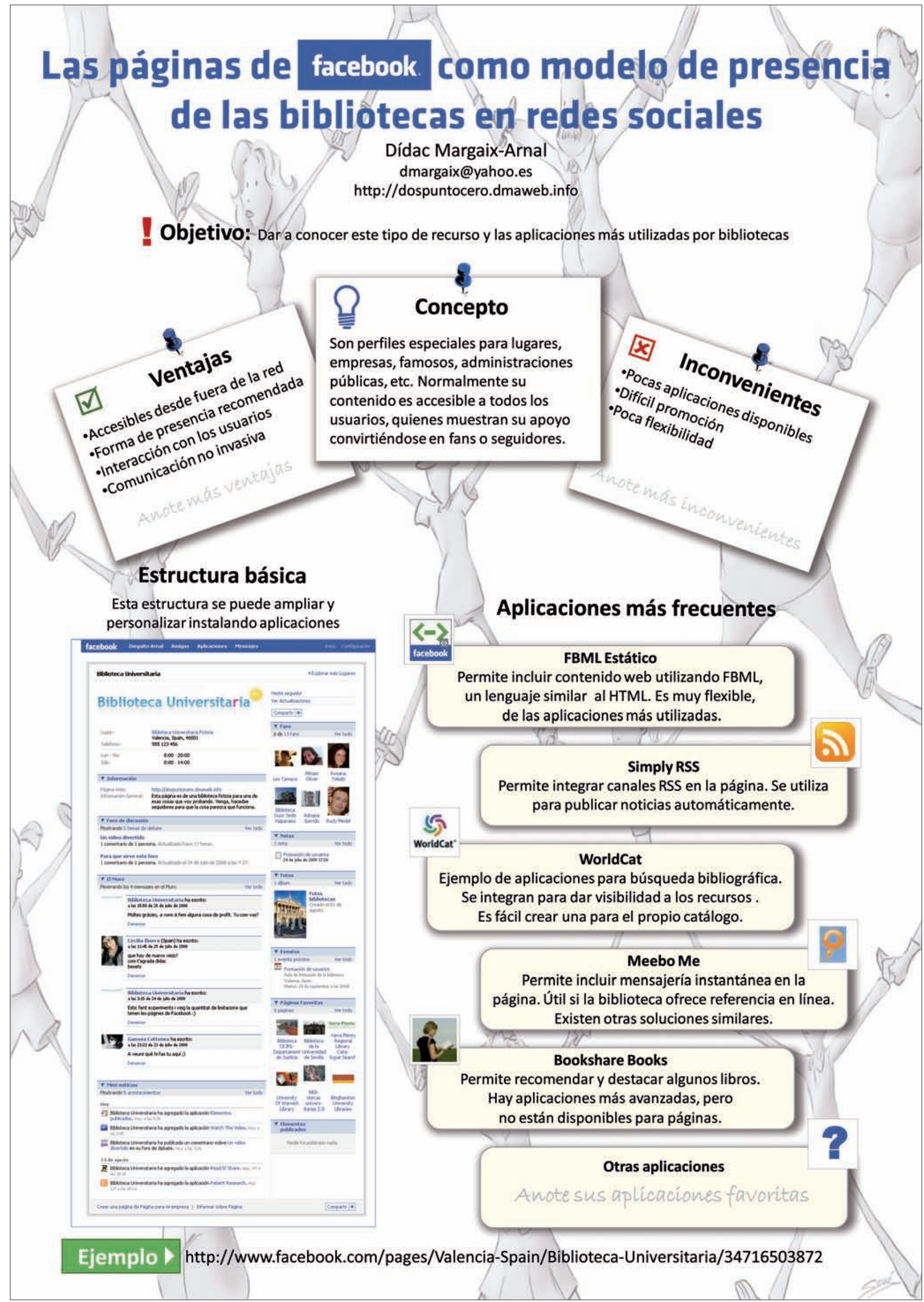

Póster presentado en el congreso "Tecnología aplicada: innovación en los servicios bibliotecarios" organizado por la Red de Instituciones Mexicanas para la Cooperación Bibliotecaria (Grupo Amigos) y celebrado en Cholula (Puebla) del 4 al 6 de septiembre de 2008 


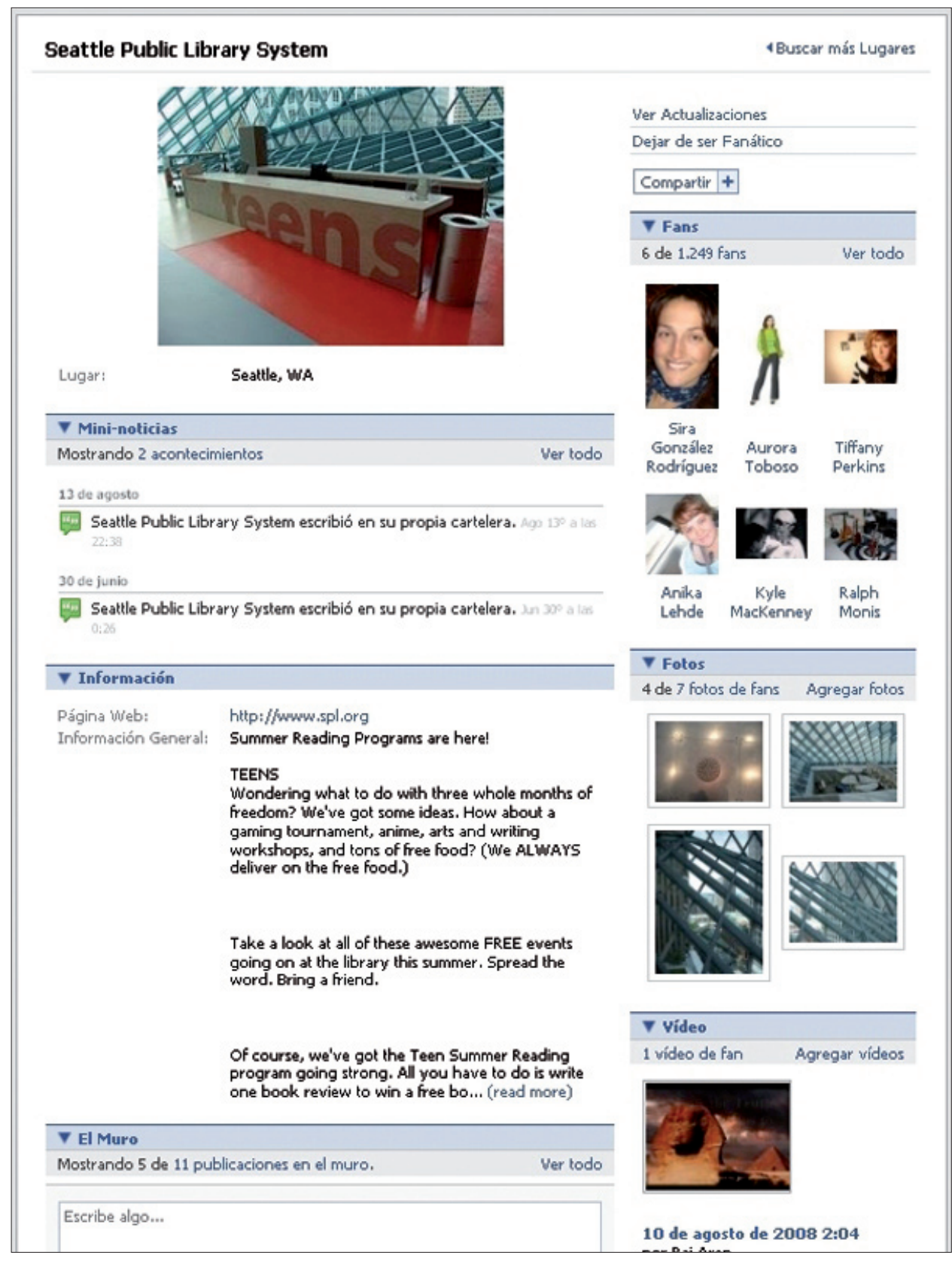

Página en Facebook de la Seattle public library. Está orientada a sus usuarios jóvenes cretar el tipo de página que se desea crear. Es conveniente seleccionar "Local" y que dentro de sus opciones "Biblioteca/Edificio público”, se añada el nombre de la biblioteca y se inicie la edición de la página. Lo más habitual es incluir una fotografía o el logotipo de la biblioteca, los datos básicos de contacto, horario, etc. Una fotografía del interior con usuarios dará una imagen mucho más cercana y viva que un logotipo o una fotografía del exterior del edificio.

La persona que cree la página quedará registrada como administrador y puede invitar a otros contactos a ser también administradores de la misma, que en definitiva son los que pueden eliminar el contenido no apropiado generado por los fans.

\subsection{Aplicaciones más fre- cuentes}

Existen algunos trabajos sobre las aplicaciones Facebo$o k$ más destacadas para las bibliotecas (Kille, 2007; Kroski, 2007; Baumann, 2008; Margaix-Arnal, 2008).

Las aplicaciones preinstaladas en una página son: tear que una página Facebook sea el sitio oficial de la biblioteca.

\section{"Las páginas de Facebook se constituyen en pequeñas sucursales digitales de la biblioteca"}

\subsection{Creación de la página}

Es algo relativamente sencillo. Es suficiente con que un usuario de Facebook visite cualquier página y pulse el enlace "Crear una página para mi empresa". Al pulsar el enlace aparece una nueva pantalla para con-
- El muro. Está pensado para que los usuarios puedan escribir mensajes a los que se les quiere dar cierta permanencia.

- Foros de discusión. Imprescindible para que los usuarios tengan dónde expresarse o conversar con la biblioteca y con otros usuarios. Se ha de supervisar su contenido para evitar conflictos, mensajes inapropiados $\mathrm{y}$ responder a las preguntas que realicen los usuarios.

- Compartir vídeos y fotografías. Permite tanto a la biblioteca como a los fans de la página publicar este tipo de materiales.

- Eventos. Útil para anunciar actividades organizadas por el centro e invitar a los contactos a participar en el evento, a los que pueden confirmar o no su asistencia. 


\section{Aplicaciones más utilizadas para páginas Facebook}

- Fbml estático.

http://www.facebook.com/apps/

application.php? id $=4949752878$

- My html.

http://www.facebook.com/apps/

application.php? id =5577282239

- Simply RSS.

http://www.facebook.com/apps/ application.php?id=491559971 1

- WorldCat.

http://www.facebook.com/apps/

application.php?id =7644880307

- CiteMe.

http://www.facebook.com/apps/

application. . php? $\mathrm{id}=23927330784$

- JStore.

http://www.facebook.com/apps/

application.php? id =2412474777

- PubMed.

http://www.facebook.com/apps/

application. php? id $=14524630141$

- Dialnet.

http://www.facebook.com/apps/

application.php? id $=18207183030$

- Catálogo colectivo de Rebiun.

http://www.facebook.com/apps/

application.php?id $=24467694387$

- MeeboMe.

http://www.facebook.com/apps/

application.php? id $=4900043878$

- Bookshare Books.

http://www.facebook.com/apps/

application.php?id=2395952879

- Notas. Ofrece la posibilidad de publicar noticias, textos, etc. Puede actualizarse automáticamente con un canal RSS.

- Las páginas también tienen disponible un interfaz de administrador, un módulo de estadísticas y una herramienta para comunicar actualizaciones a los fans o seguidores.

Otras aplicaciones que suelen utilizase para mejorar los contenidos son:
- Fbml estático. Se trata de una aplicación desarrollada por Facebook que inserta código $\mathrm{fbml}$, es decir, Facebook markup language, una versión de html adaptada por Facebook. Permite incluir varias cajas de contenido como si fueran distintas aplicaciones. Es la aplicación más versátil y utilizada. Se puede usar para insertar formularios de búsqueda, enlaces recomendados, etc. Otra aplicación similar es My html.

- Simply RSS. Permite agregar canales RSS, con lo que es posible mantener la página actualizada permanentemente con las noticias de la biblioteca o el contenido de algún blog, siempre que estén disponibles en algún lenguaje de sindicación de contenidos.

- Aplicaciones de búsqueda. Existen numerosas utilidades de búsqueda bibliográfica. Las más avanzadas son WorldCat y CiteMe, de la OCLC. También las hay para JStore y PubMed y en un ámbito más cercano otras no oficiales para Dialnet y el Catálogo Colectivo de Rebiun. Muchas bibliotecas han optado por crear utilidades de búsqueda en sus catálogos bibliográficos. Algunos autores han publicado los códigos y las instrucciones que permiten crear aplicaciones de este tipo (McCarthy, 2007).

- MeeboMe. Permite insertar una pequeña ventana de chat o mensajería instantánea.

- Bookshare Books. Presenta las cubiertas de libros recomendados por la biblioteca. Existen aplicaciones más avanzadas y sencillas de utilizar, pero sólo están disponibles para perfiles, no para páginas.

- Páginas favoritas. Establece relaciones entre distintas páginas Facebook, lo cual permite por ejemplo presentar de forma conjunta todas las páginas de las distintas sucursales de un sistema bibliotecario, otras bibliotecas especializadas en el mismo campo temático, etc.

En el directorio de Facebook se pueden encontrar otras muchas, las citadas anteriormente son sólo a modo de ejemplo. Cada centro deberá analizar sus

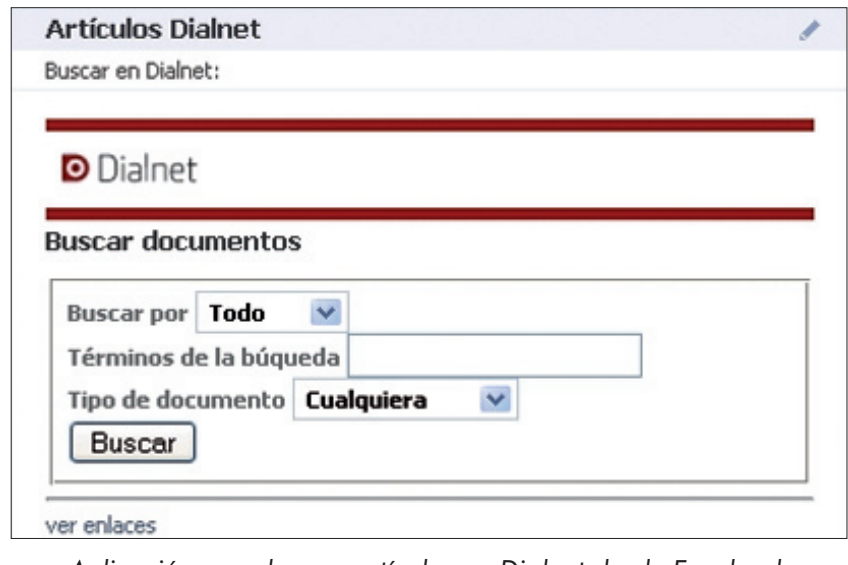

Aplicación para buscar artículos en Dialnet desde Facebook 
necesidades, cómo utilizan esta red social sus usuarios y su estrategia de comunicación para crear el servicio más ajustado a sus necesidades.

\subsection{Ejemplos de páginas de bibliotecas}

Entre las bibliotecas más relevantes que han optado por este tipo de servicio están la British Library, la Seattle Public Library y la Iowa City Public Library. Hay ya más de 150 bibliotecas universitarias que disponen de su página en Facebook, entre las que se pueden encontrar: University of Portsmouth Library, Binghamton University Libraries, Lillian Goldman Law Library, Yale Law School, David Wilson Library-University of Leicester y University of Warwick Library.
En nuestro contexto los centros que han apostado por acercarse a sus usuarios utilizando las páginas de Facebook son la Biblioteca de la Universidad de Sevilla, la Biblioteca del Centre d'Estudis Jurídics i Formació Especialitzada (Cejfe) del Departament de Justícia de la Generalitat de Catalunya y la de 1'Escola Politècnica Superior d'Enginyeria de Vilanova i la Geltrú (Epsevg) de la Universitat Politècnica de Catalunya. Algunas de estas bibliotecas mantienen paralelamente su página y un perfil que les permite una mayor interacción con los usuarios.

Para ilustrar este trabajo se ha creado dentro de Facebook una página con el nombre genérico de "Bi-

\section{Algunas páginas Facebook de bibliotecas}

- British Library.

http://www.facebook.com/pages/The-British-Library/8579062138

- Seattle public library.

http://www.facebook.com/pages/Seattle-WA/Seattle-Public-Library-System/751 1741338

- lowa City public library.

http://www.facebook.com/pages/lowa-City-IA/lowa-City-Public-Library/6184503578

- University of Portsmouth Library.

http://www.facebook.com/pages/Portsmouth-United-Kingdom/University-of-PortsmouthLibrary/8313376841

- Binghamton University Libraries.

http://www.facebook.com/pages/Binghamton-NY/Binghamton-University-Libraries/7266047455

- Lillian Goldman Law Library, Yale Law School.

http://www.facebook.com/pages/New-Haven-CT/Lillian-Goldman-Law-Library-Yale-Law-

School/14518877231

- David Wilson Library-University of Leicester.

http://www.facebook.com/pages/Leicester-United-Kingdom/The-David-Wilson-Library-Universityof-Leicester/10731483343

- University Of Warwick Library.

http://www.facebook.com/pages/Coventry-United-Kingdom/University-Of-Warwick-

Library/6168162503

- Biblioteca de la Universidad de Sevilla.

http://www.facebook.com/pages/Sevilla-Spain/Biblioteca-de-la-Universidad-de-

Sevilla/13323557934

- Biblioteca del Cejfe.

http://www.facebook.com/pages/Barcelona-Spain/Biblioteca-CEJFE-Departament-deJusticia/12147474754

- Biblioteca de L'Epsevg. http://www.facebook.com/pages/Vilanova-I-La-Geltru-Spain/Biblioteca-de-IEPSEVG/32754587255

- Biblioteca Universitaria.

Página de una biblioteca ficticia realizada para ilustrar este trabajo.

http://www.facebook.com/pages/Valencia-Spain/Biblioteca-Universitaria/34716503872 
blioteca Universitaria", donde se han instalado diversas aplicaciones y se han seleccionado como favoritas numerosas páginas de bibliotecas que pueden servir de ejemplo.

\subsection{Promoción de la página}

Los sistemas anti-spam de Facebook dificultan la promoción de la página y la comunicación con los usuarios. La recomendación es promocionarla utilizando su sistema de anuncios contextuales a los que denomina Social Ads (Vander Veer, 2008). El servicio es de pago y permite crear los propios anuncios y perfilar la campaña. Algunos bibliotecarios que lo han utilizado han relatado su satisfacción con el servicio y los resultados ${ }^{3}$.

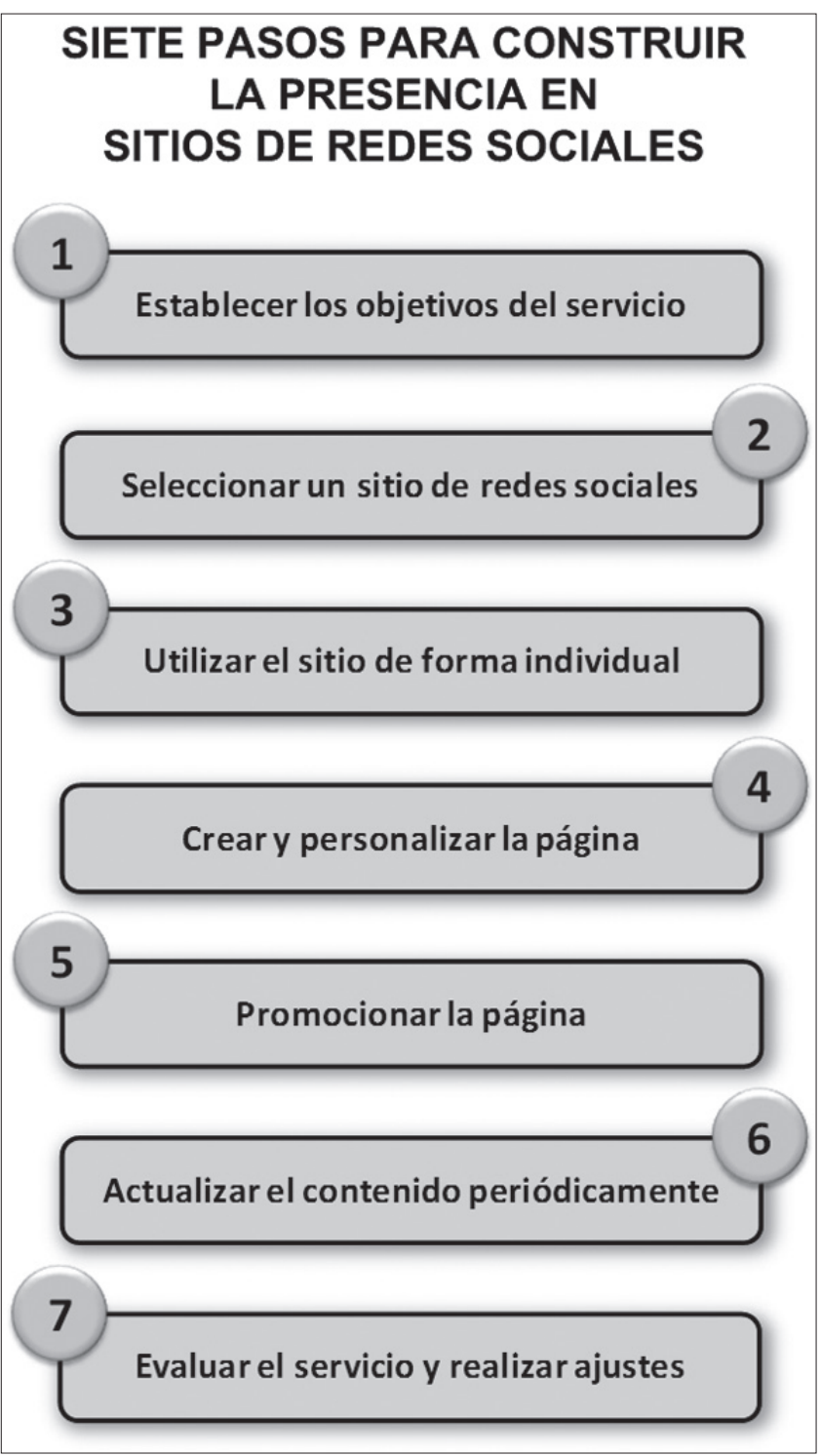

Pasos esenciales para construir la presencia de la biblioteca en los sitios de redes sociales

Para el marketing, Holzner (2008) recomienda, además de suscribir ciertos Social Ads, realizar las siguientes acciones:
- Publicitar la página desde el propio perfil.

- Darla a conocer en varios grupos afines, incluso si es posible contactar con los administradores para que consideren la posibilidad de enviar un mensaje a los miembros del grupo con la página.

- Enviar un mensaje a amigos y listas de correo.

Específicamente para bibliotecas, Behrens (2008) propone las siguientes acciones:

- Crear un link a la página Facebook desde la web de la biblioteca.

- Citar la página al final de las sesiones de formación.

- Promocionar todas las actividades de la biblioteca como "Eventos" en la página.

- Escribir cada semana consejos de búsquedas en el foro de discusión de la página o en su muro.

- Mantener y actualizar álbumes de fotos con imágenes interesantes.

- Organizar un concurso trivial mensual sólo para fans de la página.

No se pueden dejar de lado las tradicionales (o innovadoras) campañas de marketing: incluir una noticia en la web de la universidad, incluir el url en las firmas de correo electrónico del personal de la biblioteca, carteles, etc.

La campaña de promoción más conocida de una página Facebook la realizó la biblioteca de la Aurora University $^{4}$ al sortear entre todos los usuarios que se hicieran fan dos entradas para una ópera que se representaba en la ciudad, lo cual dio buenos resultados.

\section{Organizar eventos en Facebook}

Muchas bibliotecas utilizan la función "Eventos" para organizar ciertas actividades, como Librarian with a latte (Frierson, 2007). Esta iniciativa consiste en que el bibliotecario referencista, un día a unas horas determinadas, atiende las consultas de los alumnos en la cafetería. Esto ya resulta innovador por sí mismo y todo un ejemplo de cómo acercarse al usuario. Pero para promocionar la actividad se crea un evento en $\mathrm{Fa}$ cebook y se invita a los estudiantes a los que va dirigido el servicio 5 .

\section{Conclusiones}

Con independencia de los peligros que los SRS puedan presentar, es importante ser conscientes de su grado de aceptación entre nuestros usuarios. Una vez que ellos han decidido pertenecer a alguna red social, la biblioteca puede hacer dos cosas: formarles para evitar 


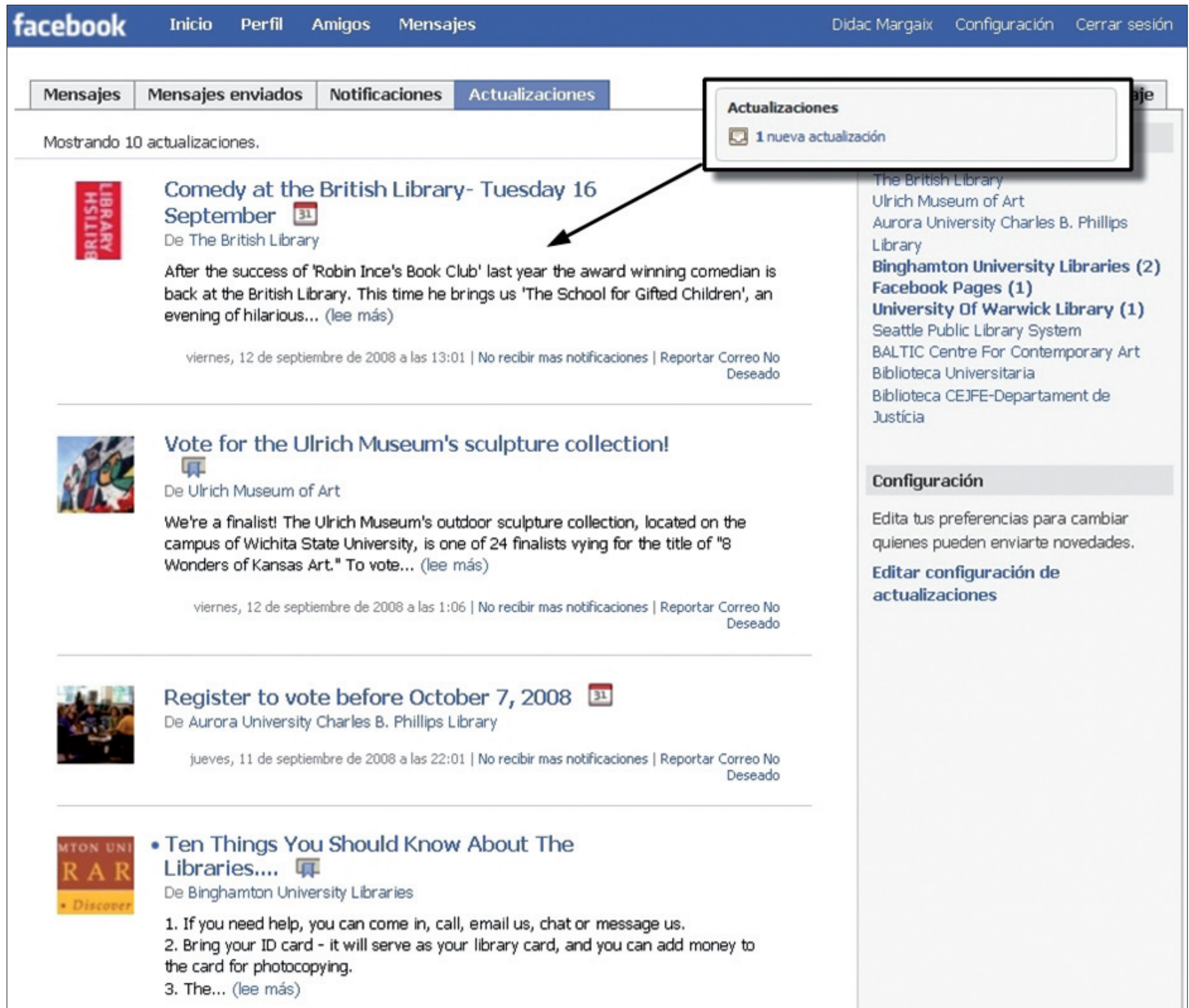

Las actualizaciones son la única forma que tiene la página de comunicarse con sus fans. En la imagen puede observarse el histórico de actualizaciones recibidas por un usuario y la imagen del aviso que le alerta de una nueva.

riesgos con respecto a su privacidad y estar presentes para ofrecer sus servicios.

La presencia de las bibliotecas en los SRS se ha de plantear como un simple "estar ahí", una puerta de acceso, un punto de inicio de conversación, una pequeña sucursal en la plataforma o una especie de acceso rápido a los principales elementos de la biblioteca. Las páginas son el elemento que mejor plasma estos objetivos dentro de Facebook.

Antes de entrar a formar parte de un SRS cada biblioteca deberá reflexionar sobre dos cuestiones fundamentales: los SRS que utilizan sus usuarios y el papel que la biblioteca puede desempeñar en ese sitio.

Si bien en la actualidad el número de usuarios españoles en Facebook es menor que en otros países, parece conveniente que los profesionales de la información vayan adquiriendo habilidades tecnológicas y sociales en este tipo de herramientas.

\section{"La presencia de las bibliotecas en los sitios de redes sociales se ha de plantear como un simple 'estar ahí'”}

Las redes sociales son algo más que una moda, como afirma Sonia Fernández (2008) "no estamos frente a un fenómeno pasajero, sino que vemos en estos sitios una respuesta a esta nueva necesidad de comunicación del internauta de hoy". ¿No es precisamente a estos internautas a quienes consideramos nuestros usuarios?, ¿no es a ellos a quien queremos asesorar en el mundo de la información?, ¿no es con ellos con quienes pretendemos conversar?

La pregunta ya no puede ser si se debe participar o no en los sitos de redes sociales; la pregunta es cómo y dónde. Es momento de reaccionar. 


\section{Notas}

1. Consultado en: 17-09-08.

http://www.alexa.com/site/ds/top_sites? $c c=E S \& t s \_$mode $=$country\&lang $=$ none

2. Consutado en: 17-09-08.

http://www.google.es/trends

3. Véase el testimonio de K. Jane. Consultado en: 17-09-08.

http://www.flickr.com/photos/kjane/2195853552/

4. En la página Facebook de esta biblioteca se puede consultar el evento. Consultado en 17-09-08.

http://www.new.facebook.com/pages/Aurora-IL/Aurora-University-Charles -B-Phillips-Library/8769270978

5. Se puede consultar el evento en Facebook organizado por Jesús Alonso-Regalado en la State University of New York-Albany. Consultado en: 17-09-08.

http://www.new.facebook.com/event.php?eid=10780762997\&ref=share

\section{Bibliografía}

Axelsson, Anna-Stina. "Libraries, social community sites and Facebook". En: Scandinavian public library quaterly, 2008, v. 41, n. 2. Consultado en: 17-09-08.

http://www.splq.info/issues/vol41_2/08.htm

Arroyo, Natalia. "Bibliotecas y redes sociales: una cuestión de visibilidad". En: IV Congreso nacional de bibliotecas públicas, 2008(a), [en prensa].

Arroyo, Natalia. "El bien abastecido mercado de los sitios de redes sociales". En: Web social para profesionales de la información, 2008(b). Consultado en: 17-09-08.

http://comunidad20.sedic.es/?p=177

Baumann, Michael. "Facebook apps: deeper social networking”. En: Infor mation today, 2008, v. 25, n. 5, pp. 1-46.

Behrens, Jennifer L. "About Facebook: change at the social-networking juggernaut creates new opportunities for law library outreach". En: AALL spectrum, 2008, v. 12, n. 6, pp. 14-17. Consultado en: 17-09-08. http://www.aallnet.org/products/pub_sp0804/pub_sp0804_Facebook.pdf

Boyd, Danah M.; Ellison, Nicole B. "Social network sites: definition, history, and scholarship". En: Journal of computer-mediated communication, 2007, v. 13, n. 1, art. 11. Consultado en: 17-09-08.

http://jcmc.indiana.edu/vol13/issue1/boyd.ellison.html

Charnigo, Laurie; Barnett-Ellis, Paula. "Checking out Facebook.com: the impact of a digital trend on academic libraries". En: Information technology and libraries, 2007, v. 26, n. 1, pp. 23-34. Consultado en: 17-09-08. http://www.lita.org/ala/lita/litapublications/ital/262007/2601mar/charnigo. $p d f$

Ellison, Nicole B.; Steinfield, Charles; Lampe, Cliff. "The benefits of Facebook 'friends': social capital and college students' use of online socia network sites". En: Journal of computer-mediated communication, 2007, v. 12, n. 4, art. 1. Consultado en: 03-10-08.

http://jcmc.indiana.edu/vol12/issue4/ellison.html

Farkas, Meredith G. Social software in libraries. Medford: Information Today, 2007, Isbn 978-1-57387-275-1.

Fernández, Sonia. "Redes sociales. Fenómeno pasajero o reflejo de un nuevo internauta". En: Telos: cuadernos de comunicación e innovación, 2008, n. 76. Consultado en: 17-09-08.

http://www.campusred.net/TELOS/articulocuaderno.asp? idarticulo= $11 \&$ rev $=76$
Frierson, Eric. "Librarian with a latte". En: Some librarian, 2008. Consultado en: 17-09-08.

http://somelibrarian.blogspot.com/2007/04/librarian-with-latte.html

Fumero, Antonio; García-Hervás, José-Miguel. "Redes sociales: contextualización del fenómeno dos-punto-cero". En: Telos: cuadernos de comunicación e innovación, 2008, n. 76. Consultado en: 17-09-08.

http://www.campusred.net/TELOS/articulocuaderno.asp? idarticulo= $3 \&$ rev $=76$

Holzner, Steve. Facebook marketing: leverage social media to grow your business. Indianapolis, Ind.: Que, 2009, Isbn 978-0-7897-3802-8.

JISC. Student expectations study: key findings from online research and discussion evenings held in June 2007 for the Joint Information Systems Committee, 2007. Consultado en: 17-09-08.

http://www.jisc.ac.uk/media/documents/publications/studentexpectations. $p d f$

Kille, Angela. "Library Facebook applications". En: Angela's blog, 2007. Consultado en: 17-09-08.

http://angelakille.wordpress.com/2007/07/20/library-facebook-applications/

Kroski, Ellyssa. "Top ten Facebook apps for librarians". En: iLibrarian, 2007. Consultado en: 17-09-08.

http://oedb.org/blogs/ilibrarian/2007/top-ten-facebook-apps-for-librariansart-onel

Mack, Daniel et al. "Reaching students with Facebook: data and best practices". En: Electronic journal of academic and special librarianship, 2007, v. 8, n. 2. Consultado en: 17-09-08.

http://southernlibrarianship.icaap.org/content/v08n02/mack_d01.html

Margaix-Arnal, Dídac. "Aplicaciones bibliotecarias para Facebook". En: DosPuntoCero, 2008. Consultado en: 17-09-08.

http://dospuntocero.dmaweb.info/2008/07/07/aplicaciones-bibliotecariaspara-facebook/

McCarthy, Graham. Facebook and your webPac, 2007. Consultado en: 17-08-2008

http://www.ryerson.ca/library/iii/facebook.html

Miller, Sarah Elizabeth; Jensen, Lauren A. "Connecting and communicating with students on Facebook". En: Computers in libraries, 2007, v. 27, n. 8 , pp. $18-22$.

Mitchell, Eleanor; Watstein, Sarah Barbara. "The places where students and scholars work, collaborate, share and plan: endless possibilities for us!". En: Reference services review, 2007, v. 35, n. 4, pp. 521-524.

Primary Research Group. Libraries and the mega-internet sites. New York: PRG, 2008, ISBN 1-57440-096-7.

Thelwall, Mike. "Social networks, gender, and friending: an analysis of MySpace members profiles". En: Journal of the American Society for Information Science and Technology, 2008, v. 59, n. 8, pp. 1.321-1.330.

Universal McCann. Power for the people: social media tracker wave 3, 2008. Consultado en: 17-09-08.

http://www.universalmccann.com/Assets/UM\%20Wave\%203\%20Final_ 20080505110444.pdf

Vander Veer, Emily A. Facebook: the missing manual. Sebastopol, CA: Pogue Press/O'Reilly, 2008, Isbn 978-0-596-51769-4.

\footnotetext{
Dídac Margaix-Arnal, Blog DosPuntoCero. dmargaix@yahoo.es http://dospuntocero.dmaweb.info
}

\section{Próximos temas centrales}

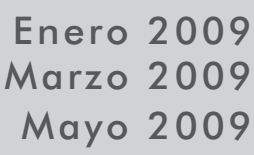

Enero 2009

Mayo 2009
Análisis y minería web

Información y movilidad: la web móvil

Documentación y medios de comunicación

Los interesados pueden remitir notas, artículos, propuestas, publicidad, comentarios, etc., sobre estos temas a: epi@elprofesionaldelainformacion.com 
DocuMenea es un sistema de noticias sobre Biblioteconomía, Documentación, Archivística,

Tratamiento de la Información, Periodismo, Internet

y Nuevas tecnologías basado en el software de Menéame.

No estés ni un día más sin leer las novedades y votar las que

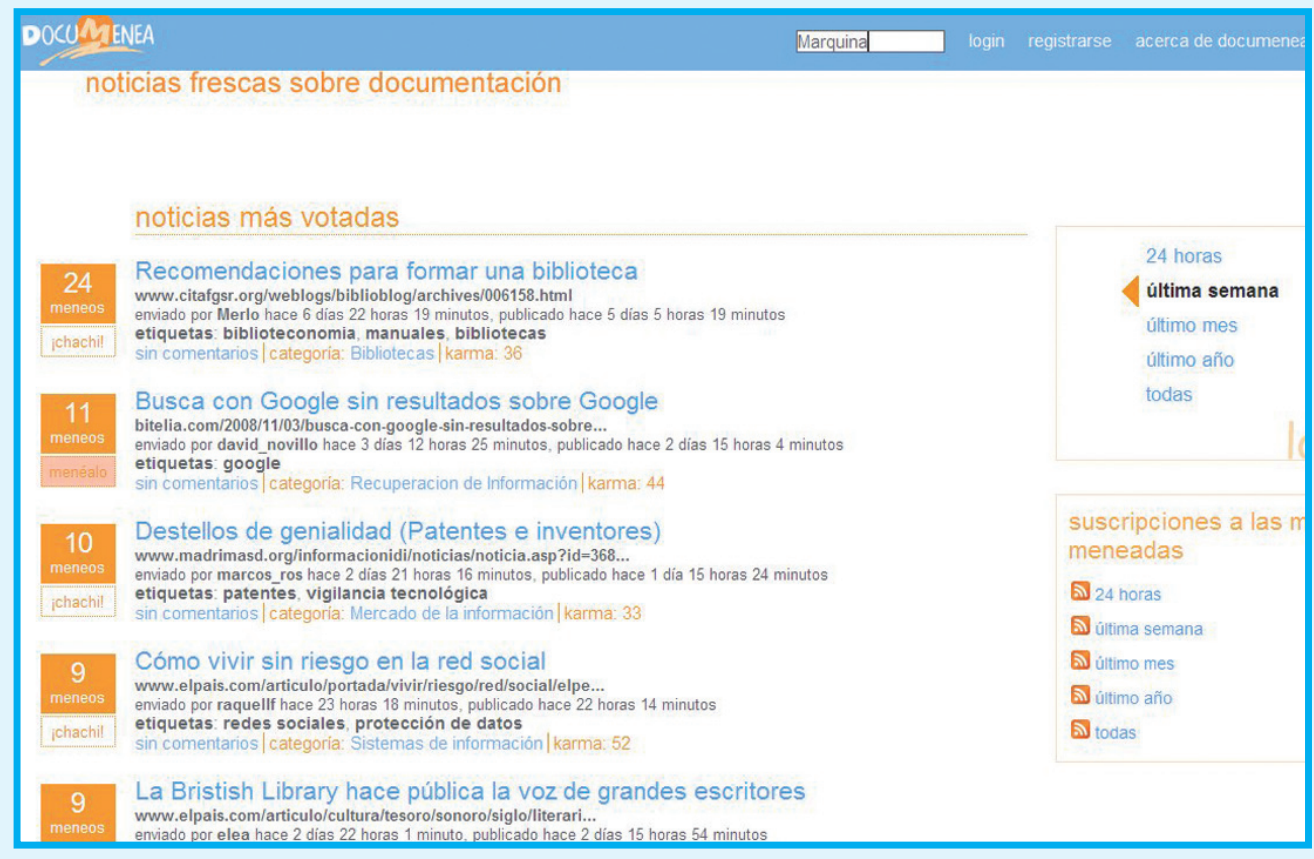
consideres importantes para hacerlas más visibles:

\section{http:/ / www.documenea.com}

\section{¿Sabías que $\mathbf{Z P |}$ tiene blog?}

\section{Se llama "Zona de notas"}

\section{http:/ / www.elprofesionaldelainformacion.com/ notas/}

En él puedes seguir toda la actualidad

y noticias sobre "El profesional de la información": novedades, comentarios sobre los contenidos, call for papers, reseñas de actividades, noticias sobre EPI en otros medios, etc.

\begin{tabular}{|c|c|}
\hline \multirow{4}{*}{ 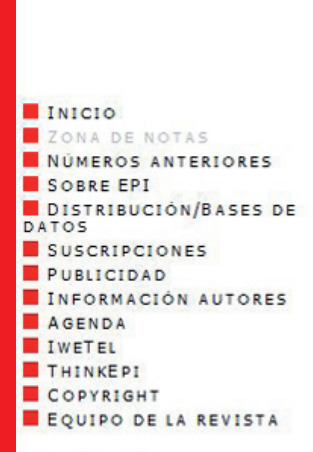 } & VO DE ZONA DE \\
\hline & $\begin{array}{l}\text { Curso en la Facultad de Medicina de } \\
\text { Salamanca (21-25 de julio de 2008) }\end{array}$ \\
\hline & Fecha de publicación: 25 Agosto 2008 \\
\hline & $\begin{array}{l}\text { El curso de verano "Biblioteca virtual en ciencias de la salud. } \\
\text { Búsqueda y recuperación de información relevante: un reto } \\
\text { para el profesional sanitario e investigador" se va consolidando } \\
\text { gracias a la buena fama que ha adquirido con sus ya } 3 \\
\text { ediciones. } \\
\text { http://sabus.usal.es/bib virtual } 3 /\end{array}$ \\
\hline $\begin{array}{l}\text { WEQUPO DE LA REVISTA } \\
\text { CONTACTO }\end{array}$ & $\begin{array}{l}\text { Los asistentes son los que mejor promoción han hecho del } \\
\text { mismo con sus comentarios entre los colegas y también en } \\
\text { blogs. }\end{array}$ \\
\hline 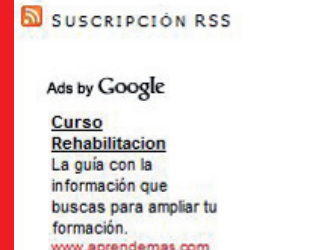 & $\begin{array}{l}\text { Los organizadores: Helena Martín- } \\
\text { Rodero, jefa de Area Biosanitaria } \\
\text { de la Biblioteca de la Universidad de } \\
\text { Salamanca, y Julio Alonso-Arévalo, } \\
\text { jefe de la Biblioteca de la Facultad } \\
\text { de Documentación y Traducción. }\end{array}$ \\
\hline
\end{tabular}

